\title{
Aldo Leopold y la Ética de la Tierra
}

\author{
Teresa Kwiatkowska \\ Universidad Autónoma Metropolitana-Iztapalapa \\ kwiat@xanum.uam.mx
}

Me parece inconcebible que una relación ética con la tierra pueda existir sin amor, respeto y admiración por la tierra,

y una alta consideración por su valor.

Desde luego que con valor me refiero a algo mucho más amplio que el mero valor económico; quiero decir, valor en el sentido filosófico.

Aldo Leopold

Está de moda señalar minuciosamente el desmedido impacto que los seres humanos hemos infligido al ambiente natural alterándolo de maneras, en su mayoría, destructivas. Si bien la preocupación por los efectos nocivos de la actividad humana en el mundo natural no se limita a la época moderna, es sólo en las últimas décadas que se ha reconocido ampliamente la gravedad de los problemas ambientales, iniciándose así la búsqueda de soluciones posibles. Al mismo tiempo es preciso recordar que nosotros no podemos vivir sin domesticar o humanizar en gran medida nuestro entorno. Moldeamos el ambiente a través de decisiones individuales y colectivas, que se toman en concordancia con diversos requerimientos 
y criterios de valor. Algunos de éstos son precisamente de orden económico, otros tienden a proteger el mundo natural que habitamos; distintas perspectivas generan variadas respuestas y dan lugar a diferentes acciones. La conservación de la diversidad biológica está íntimamente ligada a casi todos los aspectos de la vida humana. Sin embargo, la progresiva desaparición de los bosques, ante todo pluviales, la pérdida de la biodiversidad en razón de la destrucción de su hábitat y el posible cambio climático no provocan una atención y acción proporcionales a la seriedad de los problemas.

Aquí conviene recordar que todas las elecciones relacionadas con el mundo natural están íntimamente ligadas con preferencias y deseos humanos. En general, la mayoría de las personas llegan a preocuparse por el daño infringido al ambiente sólo cuando lo perciben como un peligro para los propios intereses humanos. Si, por ejemplo, el factible calentamiento global no abrigara consecuencias nocivas para nosotros, probablemente su riesgo se vería acompañado por la indiferencia. Para no caer en confusiones, hay que aclarar que la perspectiva antropocéntrica es una condición ontológica del ser humano. No podemos entender el mundo y la vida que alberga desde otra perspectiva que no sea la humana. Toda la realidad está sujeta a nuestras propias interpretaciones, a una humanización. Nada tiene sentido, nada tiene un fin sin que el hombre conceda un valor a una cosa o a un ser viviente. Somos también nosotros quienes establecemos derechos y obligaciones. En fin, los intereses del ser humano dominan las doctrinas éticas de la filosofía moral occidental.

Igualmente, importa recordar que, a lo largo de la historia y hasta mediados del siglo xIX, ha dominado la idea de que el mundo natural constituye una adversidad si se deja tal cual frente a los recursos que su cultivo puede procurar. La na- 
turaleza indómita fue percibida como hostil, salvaje, incluso malvada. En la historia de la conservación americana, los Románticos (Ralph Waldo Emerson, Henry David Thoreau y John Muir) fueron los primeros escritores que abandonan la idea de la naturaleza como un lugar peligroso y horrible del tiempo de los pioneros, para convertirla en un entorno digno de ser admirado, en un signo de identidad, en la reivindicación de un espacio vital para el ser. La naturaleza salvaje ya no es un lugar a domesticar, sino un espacio a preservar. Muir (1838-1914), uno de los más importantes e influyentes naturalistas de su época, concibe una visión casi religiosa de la naturaleza y critica una superflua civilización materialista y utilitarista, que aleja al hombre moderno del entorno natural. Esta sensibilidad romántica y el afán racional de preservar extensas áreas naturales llevaron a la creación de parques naturales nacionales, como los de Yellowstone y Yosemite. El primero fue creado por el decreto del presidente Grant, en 1872, y, el segundo, por Abraham Lincoln en 1864. Por otra parte, los conservacionistas como Gifford Pinchot y sus seguidores, herederos de la Ilustración, presuponían que los imperativos biológicos fundamentales deberían cimentar las decisiones ambientales, y abogaban por armonizar las necesidades económicas de las comunidades con los principios ecológicos para el uso prudente de la Tierra.

Fue justamente Aldo Leopold (1887-1948), académico de la Universidad de Wisconsin, y un especialista en el manejo de recursos forestales, quien reunió las dos tendencias en una visión que ha afectado la conservación contemporánea de los recursos naturales de manera más profunda y duradera. Fue uno de los profesores de la ecología silvestre que fundaron la primera área protegida de la naturaleza indómita, y pionero en restauración de las comunidades de las plantas nativas 
(ver Meine, 1988). Cuando un joven guardabosques vio por la primera vez el paisaje de Gila (al suroeste de Nuevo México), en 1915, quedó impresionado por la naturalidad de esta tierra de majestuosas y escarpadas montañas, profundos cañones y hermosísimo bosque. A consecuencia de esto, dedicó la siguiente década de su vida para la designación de Gila como área silvestre, logrando su propósito en junio de 1924, cuando el Servicio Forestal de los Estados Unidos designó a una porción del aquel bosque (Gila National Forest) como la primera área silvestre oficial del país. Años después, durante uno de sus viajes para cazar allá, Leopold reflejó lo siguiente:

\begin{abstract}
Alcanzamos un viejo lobo a tiempo para ver el verde fuego decreciendo en sus ojos. Fue en este momento cuando me di cuenta, y lo recordé durante toda mi vida, que hubo algo nuevo, desconocido en sus ojos [...] algo solamente personal de ella y de la montaña. Estuve joven e impetuoso en estos días; pensé que menos lobos y por tanto más venados, crearían un paraíso para los cazadores. Pero viendo morir este verde fuego, sentí que ni el lobo y la montaña concordaron con mi fantasía.
\end{abstract}

Las ideas tempranas sobre la conservación las formuló en el ensayo titulado: «Some Fundamentals of Conservation in the Southwest», escrito en 1924. En otro artículo elaborado en el mismo año, Leopold señaló una relación bastante problemática entre el pastoreo de vacas, la quema de bosques y praderas, con la erosión del suelo (ver Leopold, 1924). También, hay que recordar que en 1918, en un artículo poco conocido, indicó los peligros de la mezcla genética de los peces nativos acostumbrados a los hábitats específicos (ver Leopold, 1918; 101-102). 
Sin embargo, ha sido la publicación póstuma de su libro A Sand County Almanach and Sketches Here and There lo que le llevó a la fama. Este libro tiene dos partes claramente diferenciadas. En la primera, Leopold relata, con sencillez y afecto, magníficas experiencias de sus vivencias en el campo, acorde al devenir de las estaciones: la llegada de la primavera, las diferentes clases de aves (era un experto y reputado ornitólogo), la vida en el bosque, la nieve, el viento, los incendios, el suave movimiento de las plantas en las grandes praderas, la nevada en invierno. En la segunda parte del libro desarrolla su pensamiento, apuntando unas ideas sumamente simples, pero de una lógica tajante en la aceptación de los componentes biológicos como bases fácticas de una ética idónea para los sistemas naturales y entornos humanos.

Su corto pero célebre escrito La ética de la tierra resalta la trascendencia de la ética ambiental en el manejo del entorno natural, encabezando así los credos nuevos de nuestra época. La intención general de Leopold fue poner de manifiesto que la tierra, desde el punto de vista moral, no debiera ser considerada como una propiedad sino como una comunidad a la cual pertenecemos todos. Después de varios años de trabajo de campo en el manejo de bosques, llegó a la conclusión de que los puros reglamentos y las políticas conservacionistas, por más oportunos que sean, no bastan para disminuir la destrucción del ambiente. Es necesaria una «ética de la tierra» que incluiría a los ecosistemas y sus habitantes no humanos en la preocupación moral. Así que propuso ampliar su cobertura hasta las relaciones de la humanidad con la tierra en su conjunto, lo que implica ipso facto un profundo cambio de contenido en la normatividad tradicional. En este texto él acredita la administración de los ecosistemas no sólo con emociones y principios morales, sino también con un saber 
científicamente verificable. Su nuevo enfoque que reconoce el valor de toda la vida, humana y no humana, en una visión integrada del mundo, convierte al libro A Sand County Almanac en la biblia del movimiento ambientalista mundial. Inclusive varias corrientes de la ética ambiental y de las políticas de conservación actuales hunden sus raíces en las ideas expresas de Leopold. Allá escribe: «Una ética de la tierra cambia el papel del Homo sapiens de conquistador de la Tierra por el de mero miembro y ciudadano de ella. Ello implica respeto para sus semejantes y también para la comunidad como tal». De hecho, Leopold puede ser considerado como uno de los visionarios ecológicos cuyas ideas y sugerencias tardaron alrededor de setenta años para por fin repercutir en la definición del concepto de manejo de los ecosistemas, el cual combina los proyectos utilitarios de Pinchot con los ideales de conservación propuestos por Muir. Escribió:

[...] un sistema de conservación basado solamente en un egoísta interés económico es irremediablemente desequilibrado. Tiende a ignorar, y por lo tanto a eliminar finalmente, muchos elementos en la comunidad de la tierra que no tienen valor comercial, pero que son (hasta donde nosotros sabemos) esenciales para que ésta funcione sanamente. Asume -erróneamente, creo yoque las partes económicas del reloj funcionarán sin las partes no económicas $(1949 ; 251)$.

Cuando Aldo Leopold afirma que las políticas de conservación, apoyadas únicamente en el interés propio económico, son insensatas, todos le dan la razón. Luego, señala agudamente que la cuestión de los incentivos financieros que podrían aliviar la pobreza e indicar una alternativa viable que se aleje de las prácticas ambientalmente dañinas tiene que resol- 
verse con prontitud. Estas ideas han alterado todas las políticas ambientales posteriores más allá de su país nativo; en la toma de decisiones la humanidad empezó a compartir la importancia ética con su entorno natural. La conservación del mundo natural, aparte de las consideraciones científicas y económicas, había adquirido una perspectiva ética.

A diferencia de las tendencias religiosas o místicas que frecuentan los debates morales acerca de las relaciones de los humanos con el mundo natural, Leopold cimentó su reflexión en las ciencias biológicas de su tiempo. En la introducción a su libro, escribió: «Que la tierra sea una comunidad constituye el concepto básico de la ecología, pero el que se deba amar y respetar a la tierra es una extensión de la ética» (1996; 236). Parte del concepto de «comunidad biótica» y considera al ser humano una parte intrínseca de la naturaleza. Señala los pasos de la evolución ética desde la perspectiva de la preocupación por la excelencia moral personal, pasando por las relaciones que se dan entre el individuo y la sociedad, hasta la relevancia de los lazos con nuestro medio natural. En la Ética de la Tierra escribe: «La primera ética se ocupó de la relación entre los individuos [...] Ampliaciones posteriores trataron de la relación entre el individuo y la sociedad [...] Hasta ahora no hay una ética que se ocupe de la relación del hombre con la tierra y con los animales y plantas que crecen sobre ella [...] La extensión de la ética a este tercer elemento en el ambiente humano es [...] una posibilidad evolutiva y una necesidad ecológica» $(1998 ; 62)$.

Bajo la influencia de Charles Darwin, considera a la ética como un proceso que forma parte de la evolución ecológica y «una limitación de la libertad de acción en la lucha por la existencia». La tierra de su discurso es un nombre genérico para el medio ambiente y comprende, en sus palabras, «los 
suelos, las aguas, las plantas y los animales» (1998; 62). Dentro de esta reflexión, el humano deja de ser dueño de la naturaleza y también un intruso que perturba los ritmos del mundo natural, para convertirse en integrante de la "comunidad biótica». En su cuento sobre el «roble nudoso», Leopold escribe: «Así, quien posee un viejo roble nudoso posee más que un árbol. Posee un biblioteca histórica y un asiento exclusivo en el teatro de la evolución» $(1996 ; 30)$. Aquí hay que resaltar que Leopold no cuestiona en ningún momento la ética tradicional; sólo propone su extensión a las relaciones de la humanidad con la Tierra y, por tanto, un cambio de contenido en la normativa tradicional. Toma en cuenta las diferencias entre humanos y no humanos, pues a ambos les otorga consideración moral, si bien les da un trato distinto. Se centra en la interrelación de todas las formas de vida, incluyendo a los componentes abióticos (no vivientes) del entorno. De esta forma, propugna un desplazamiento del centro del interés moral desde lo momentáneo e individual hacia lo temporalmente más duradero y hacia un círculo más amplio de bienestar. Su ética se convierte en la expresión axial de una perspectiva centrada en la naturaleza. Queda claro que cada extensión de la esfera ética significa la entrada de un nuevo miembro a la comunidad moral, un miembro que, considerado de una manera no instrumental, es digno de respeto y consideración moral. Es, por tanto, que la ética de la tierra articula valores que compiten con nuestras preferencias actuales, puesto que casi toda la tradición ética se restringe al mundo de la cultura humana, donde todo lo demás, como la flora, la fauna y, más en general, la Tierra, no cumplen más que una función propiamente instrumental. La filosofía moral tradicional no promueve ninguna obligación moral directa en relación con los ecosistemas, las plantas o los animales. Una de las carac- 
terísticas más distintivas de la presente filosofía ambiental, heredada del pensamiento de Leopold, es el esfuerzo por desarrollar una teoría del valor no antropocéntrica, que defina el bien independientemente de cualquier cualidad humana, un bien cuyas propiedades se encuentren en un mundo terrestre no humano. Sin embargo, hay que subrayar que el propósito de la "ética de la tierra» no consiste tanto en atribuirle un valor «intrínseco» a los ecosistemas, como lo sugieren las corrientes no antropocéntricas de la ética ambiental, sino más bien en reconocer los múltiples valores comunitarios y buscar la integración de valores pluralistas en múltiples niveles. Esto ofrece una base potencial para proteger y conservar la diversidad cultural y biológica de maneras socialmente justas y económicamente eficientes.

En consecuencia, Leopold sugiere la existencia de una relación íntima e indisoluble entre el bienestar humano y el de las otras especies biológicas, invitándonos a reflexionar sobre nuestras actitudes hacia el mundo, un entorno del que formamos parte. Su visión nos conduce a considerar el bienestar de la naturaleza como parámetro del carácter moral de nuestras acciones. En términos prácticos, él no se opone a las actividades humanas necesarias para producir alimentos, utilizar los recursos o diseñar el paisaje. Lo que impugna es la contaminación del ambiente y la destrucción de la biodiversidad, que recientemente cubre con su manto negro desde el material genético, hasta los ecosistemas enteros. Se trata de una ética cuya tarea no consiste en moralizar, sino en iluminar a los seres humanos acerca de quiénes somos, qué lugar ocupamos y qué hacemos. Por lo tanto, su ética de la tierra es un espejo, no un modelo. En su famoso pronunciamiento, Aldo Leopold asume que «una cosa es correcta cuando tiende a mantener la integridad, la estabilidad y la belle- 
$z a$ de la comunidad biótica; y es incorrecta cuando tiende a hacer lo contrario» (1949; 224-225).

De hecho, las hipótesis que conciben a la comunidad biótica (con toda la vida que ésta cobija) como un sujeto moral que exige respeto, así como la forma en que varios filósofos han interpretado a Leopold, redundan en propuestas éticas que asumen la independencia del valor de los sujetos biológicos como individuos o colectividades. Incluso, unos filósofos ambientales toman estos conceptos simbólicos al pie de la letra, y aceptan que ciertos rasgos objetivos de los ecosistemas como la integridad, la estabilidad y la belleza constituyen fuentes de valor intrínseco y fundamento de obligaciones morales, o, mejor dicho, el «principal aserto prescriptivo» que debería ser adoptado como un punto central de la legislación ambiental (ver Westra, 2003; 165-180). Por otra parte, John Baird Callicott, en «La ética de la tierra en nuestros días», afirma que la integridad y la estabilidad de los ecosistemas ha de entenderse metafóricamente como «salud» o «capacidad de autorrenovación» que se traduce en criterios objetivos como la diversidad de especies o la variabilidad genética (ver Callicott, 2004; 45-68). Tradicionalmente, el concepto de la integridad suele designar nuestro sentido de la totalidad y el bienestar, o la total o amplia gama de aptitudes poseídas por una persona. En el contexto estrictamente ecológico lo definen como funcionalidad de los ecosistemas, el cual se traduce en la condición de operación normal de un ecosistema cuando posee todos sus elementos funcionales operando adecuadamente, y cuando sus procesos se encuentran en condiciones estables y duraderas. No cabe duda que Leopold nos dio una idea simbólica de la necesidad de utilizar la información biológica y ecológica en la toma de decisiones sobre nuestro bioentorno. Pero hay que 
resaltar que la naturaleza resiste la imitación a través de un modelo fijo, y por tanto todas las decisiones ambientales son y serán tomadas a partir de un conocimiento incierto e incompleto. Dejando atrás dudas, preguntas y críticas que rodean el concepto de integridad en el mundo natural; es cierto que las ideas expresadas por Leopold hace muchas décadas pueden desempañar un papel crucial dentro de nuestras relaciones con el ambiente sustituyendo a las actitudes estrictamente económicas. Conocer las dimensiones biológicas de los problemas ambientales junto con nuestra integridad moral nos ayudaría, ciertamente, a escoger la mejor alternativa ecológica y social.

Oscar Wilde escribió una vez que el peor crimen del ser humano era la falta de imaginación; pues no nos compadecemos de aquellos males de los que no tenemos experiencia directa, ni de aquellos a los que no hemos asistido. Cuando todos los sistemas éticos desarrollados hasta el momento han fracasado en su intento de universalidad, surge la pregunta de por qué motivos no hemos sido capaces de encontrar una guía común para nuestras acciones. Quizás por esta razón Leopold sostuvo que "podemos ser éticos sólo en relación con algo que podemos ver, sentir, entender y amar o en lo que tenemos fe» $(1998 ; 69)$. Esta creencia suya sobre las emociones nos obliga a reflexionar y, esto es más importante, a sentir los motivos de las decisiones que tomamos diariamente. Más allá del lenguaje, nuestras emociones se configuran como colina desde la cual exploramos el mundo que nos rodea, el punto de partida hacia los otros, e incluso hacia lo más básico del mundo natural que nos rodea.

Igualmente, Leopold piensa que la protección de la naturaleza silvestre ayuda a fomentar la felicidad y el florecimiento de los seres humanos, alentándonos espiritualmente 
para ser mejores personas. En la naturaleza «vemos eternamente lo que nos salva»; nuestra recreación y disfrute de sus fenómenos y sus criaturas concuerda con un fuerte sentido moral y virtuoso. «Estoy protegiendo la selva lluviosa» se transforma en «Soy parte de la selva lluviosa y me protejo a mí mismo. Soy parte de la selva lluviosa que recientemente alcanzó la autoconciencia». Superando las ilusiones antropocéntricas, Leopold comenzó a "pensar como una montaña» (ver Sessions, 1979).

En virtud de este enfoque, se opta por la igualdad biótica según la cual todas las cosas naturales -los ecosistemas, la vida, los paisajes, los suelos, las montañas, las praderas, el conjunto del mundo natural- tienen derecho intrínseco a existir. En su visión, la presencia del valor intrínseco es independiente de cualquier razón, interés o apreciación del ser humano. De la filosofía moral de David Hume y Adam Smith, Leopold toma prestado el concepto de altruismo como fundamento de los valores y juicios morales dentro de la comunidad social. Pero, más que nada, los valores de la conservación del mundo natural se desprenden de la relación íntima del ser humano con la tierra. "La ética que complementa y guía la relación económica con la tierra presupone una "imagen mental" de la tierra como un mecanismo biótico».

Son las ciencias de la vida las que nos formulan una «imagen mental» de la naturaleza. Sin embargo, «la imagen no es un último análisis del mundo natural en sí, se trata solamente de una imagen de nuestra relación con la naturaleza» $(1976 ; 24)$, como lo indicó Werner Heisenberg, un reconocido físico de principios del siglo pasado. La ecología no proporciona secuelas éticas que puedan orientar las actitudes individuales y políticas sociales benéficas para el ambiente natural. La investigación científica del mundo natural y la búsqueda ética 
de nuestras experiencias morales son indagaciones independientes; cada una de ellas se desarrolla en diferentes terrenos de información y goza de consecuente autonomía. Pero, a pesar de que a los valores o virtudes de la ética ambiental no se llega por la vía de las ciencias de la naturaleza, éstas pueden sugerirnos un camino útil en la toma de decisiones sociales y ambientales, y ayudar a activar la más amplia voluntad social, imprescindible para conservar las diversas comunidades ecológicas. Como lo expresó Ernst Mayr, reconocido biólogo contemporáneo: «La ignorancia de los hallazgos de la biología es fundamentalmente dañina cuando los políticos están obligados a enfrentar problemas como la sobrepoblación mundial, el agotamiento de los recursos renovables y no renovables, los cambios climáticos peligrosos, el incremento de las necesidades agrícolas en el mundo entero y la destrucción de los hábitats naturales». Su opinión coincide con las ideas de nuestro autor, quien sostuvo que el juicio moral responsable debe basarse en una comprensión plena del significado de los hechos.

Leopold, muy prudentemente, define la ética como una «especie de guía para enfrentar situaciones ecológicas tan nuevas e intrincadas, o que involucren reacciones diferidas tales que la trayectoria de la conveniencia social no sea discernible para el individuo promedio» (1998; 239) o, mejor dicho, que abarcan reacciones en un futuro tan lejano que una persona común no puede predecir las consecuencias últimas de dichas circunstancias. Siguiendo sus conjeturas, la ecología y la ética deberían coparticipar en la concepción del mundo, de tal modo que podamos ver a través del impacto combinado de los resultados experimentales y los nuevos conceptos éticos. Este enfoque integrado de la relación entre la sociedad humana, la economía y los sistemas naturales, 
permite el logro de las metas sociales al tiempo que se conserva el mundo natural.

No obstante, la ética de la tierra tiene también sus problemas. Da lugar a una serie de preguntas que involucran las funciones vitales de todos los integrantes de la comunidad (seres humanos versus no humanos); el estatus, la intensidad y las fronteras temporales y espaciales de la comunidad; la clase de obligaciones que tenemos para con los demás miembros de la comunidad. A todas estas cuestiones, los ambientalistas les han dado diversas respuestas. Todavía no está claro en los sistemas legales quién tiene la obligación de preservar la biodiversidad de invaluables áreas naturales, como el bosque tropical, los pantanos y demás. Más aún, vivimos en un mundo donde millones de personas sufren desnutrición, hambre y extrema pobreza. «Sin lugar a dudas -escribe el filósofo ambiental Brian Norton- Leopold y Carson han contribuido más que nadie a configurar el pensamiento de los ambientalistas actuales, y ellos compartían la voluntad de llegar con sus argumentos a instancias políticas relevantes. Ambos estaban profundamente comprometidos con una perspectiva biocéntrica y atacaron la arrogancia humana. Sin embargo, cuando se encontraban en arenas políticas enfatizaron siempre argumentos orientados hacia el bienestar humano».

Para finalizar, hay que recordar, como lo apuntó el humanista y microbiólogo René Dubos (autor de la frase «Pensar globalmente, actuar localmente»), que: «La conservación se desprende de los valores humanos; su sentido más profundo está en el contexto y en el corazón humano. Salvar pantanos o bosques de secoya no exige una justificación biológica como tampoco lo requiere oponerse a la crueldad y al vandalismo» (ver Dubos, 1972). Y -éste es el punto primordial- nuestra 
intuición moral, la cual valora al mundo natural con todas sus criaturas, no requiere de una hipótesis científica sobre su modo de organización. Las diferentes percepciones de una maravillosa diversidad de plantas, animales y microorganismos, «allá afuera», pueden capturar la imaginación de las personas, despertando su sensibilidad y su responsabilidad moral. Es importante señalar que si los valores ambientales llegaran a ser compartidos, esto podría significar un acuerdo para negociar diferencias y conciliar nuestras necesidades y preferencias con la conservación de las riquezas biológicas que corren el riesgo de desaparecer.

\section{BIBLIOGRAFÍA}

Anderson, P. (1995). Aldo Leopold, American Ecologist. Nueva York: Franklin Watts/Grolier.

Brown, D. E. \& N. B. Carmony (eds). (1990). Aldo Leopold's Southwest. (Twenty-six early writings). Albuquerque: University of New Mexico Press. [Publicación original: Aldo Leopold's Wilderness, Stackpole Books].

Callicott, J. B. (2004). «La ética de la tierra en nuestros días». En: Valdés, M. (ed.), Naturaleza y Valor: Una aproximación a la ética ambiental (pp. 45-68), México: Fondo de Cultura Económica.

Callicott, J. B. \& E. T. Freyfogle (eds). (1999). Aldo Leopold: For the Health of the Land. (Previously unpublished essays and other writings). Washington DC: Island Press/Shearwater Books.

Callicott, J. B. (ed). (1987). Companion to A Sand County Almanac. Madison: University of Wisconsin Press. 
Dunlap, J. (1993). Aldo Leopold-Living With the Land. Nueva York: Twenty-First Century Books/Henry Holt.

Flader, S. \& J. B. Callicot (eds). (1991). The River of the Mother of God and Other Essays by Aldo Leopold. Madison: University of Wisconsin Press.

Flader, S. (1974). Thinking Like A Mountain: Aldo Leopold and the Evolution of an Ecological Attitude Toward Deer, Wolves and Forests. Columbia: University of Missouri Press.

Kwiatkowska, T. \& J. Issa (eds). (1998). Los caminos de la ética ambiental: una antología de textos contemporáneos. México: Plaza y Valdés.

Leopold, A. (1998). «La Ética de la tierra». En: Kwiatkowska, T. \& J. Issa (1998).

. (1996). A Sand County Almanac. Nueva York: Ballantine Books.

. (1949). A Sand County Almanac and Sketches Here and There. Londres: Oxford University Press,

. (1933). Game Management. Nueva York: Charles Scribner's Sons. [Reimpreso en 1986 por University of Wisconsin Press, Madison].

. (1924). «Grass, Brush, Timber, and Fire in Southern Arizona». Journal of Forestry 22 (6); 1-10. [Reimpreso en: The River of the Mother God and Other Essays. S. L. Flader \& J. B. Callicott (eds). Madison: University of Wisconsin Press, 1991].

. (1918). «Mixing trout in western waters». Transactions of the American Fisheries Society 47; 101-102.

McCabe, R. (ed). (1989). Aldo Leopold, Mentor. Madison: Department of Wildlife Ecology, University of Wisconsin. McCabe, R. (1987). Aldo Leopold, The Professor. Amherst, WI: Palmer Publications. 
Meine, C. \& R. L. Knight (eds). (1999). The Essential Aldo Leopold. Madison: University of Wisconsin Press.

Meine, C. (1988). Aldo Leopold: His Life and Work. Madison: University of Wisconsin Press.

Sessions, G. (1979). Spinoza, Perennial Philosophy and Deep Ecology. California: Sierra College.

Tanner, Th. (ed). (1987). Aldo Leopold, The Man and His Legacy. Ankeny, IA: Soil Conservation Society of America.

Westra, L. (2003). «De Aldo Leopold al Proyecto de las Áreas Silvestres: la ética de la integridad». En: Kwiatkowska, T. \& J. Issa (1998);165-180. 


\section{RESUMEN}

En este artículo se desarrollan los conceptos centrales de la ética de la tierra defendida por el filósofo norteamericano Aldo Leopold. Se defiende que la ecología y la ética deberían coparticipar en la concepción del mundo, de tal modo que los seres humanos puedan ver a través del impacto combinado de los resultados experimentales y los nuevos conceptos éticos. Este enfoque integrado de la relación entre la sociedad humana, la economía y los sistemas naturales, permite el logro de las metas sociales al tiempo que se conserva el mundo natural.

Palabras clave: Aldo Leopold; ética de la tierra; antropocentrismo; comunidad biótica; ética ambiental.

\section{ABSTRACT}

In this paper I develop the central concepts of «land ethic» advocated by American philosopher Aldo Leopold. I argue that ecology and ethics should co-participate in the conception of the world, so that humans can see through the combined impact of the experimental results and new ethical concepts. This integrated approach to the relationship between human society, economy and natural systems, allows the achievement of social goals while preserving the natural world.

Key words: Aldo Leopold; land ethic; anthropocentrism; biotic community; environmental ethics. 